\section{A ratchet made of tiny magnets}

Standfirst [max. 250 characters including spaces]

Steven T. Bramwell

Arrays of interacting magnets have long been a source of fascination. The first to be studied covered a table-top and were observed by eye [1]. The modern equivalents cover perhaps a billionth of that area and are observed - with equal precision - by marvellous techniques such as x-ray photoemission electron microscopy [2]. This impressive miniaturisation has given the arrays thermal as well as mechanical properties and has effectively turned them into a class of metamaterial. But has it brought us any closer to applications? As with the case of real materials, the first challenge is to introduce functionality and to understand the principles by which it operates. Writing in Nature Materials, Sebastian Gliga and colleagues describe a micro-magnetic array that functions like one of the most basic and useful of all devices: a ratchet [3]. Their array consists of fixed magnetic needles of sub-micron size (Fig. 1a, left). After a magnetic field is removed, the magnetisation (both of the elements and the whole array) thermally relaxes by rotating in one unique sense. How this comes about rather beautifully illustrates the concepts of symmetry and topology in two dimensions.

The functionality of the micro-magnetic array may be understood by analogy with a mechanical "ratchet and pawl". This has two key features. The first is that the ratchet wheel, when confined to rotate in two dimensions, is a chiral object. Chirality in two dimensions means that no movement within the plane can bring an object into coincidence with its mirror image. For example the letters " $b$ " and " $p$ " are mirror images in a horizontal line and hence are a chiral pair. Chirality is more important in two dimensions than in three because many objects that are achiral in three dimensions become chiral in two: the ratchet wheel and the letters $b, p$ are examples. Another common feature of two-dimensional chirality is that periodic arrays of chiral objects are often achiral when infinitely extended but chiral when finite. Consider, for example, the sequence bpbpbpbpp, and its chiral image formed by reflection in a horizontal line, pbpbpbpbpb. If the sequence is infinite then the combined reflection and shift of one space to the right or left - a "glide reflection" - will bring the two sequences into coincidence. In contrast any attempt to superimpose the two finite sequences by means of such a shift breaks down at the ends of the sequence: we end individual elements actually rotating, or just switching direction in a specific order? Can we replace it with "After a magnetic field is removed, the magnetization of the whole array thermally relaxes..." clear, yet a bit too long, and it may distract the attention of the reader from the main results of the paper. Could you shorten it, for instance by removing this sentence? 
up with bpbpbpbpbpb or pbpbpbpbpbp, where the bold letters indicate those letters that have been successfully superimposed. It is the left-over letters at end of the sequence (those not in bold) that make the finite sequence chiral.

In the micro-magnetic array of Gliga et al., something similar happens. As shown in Fig.1a, we can describe the array in terms of the repetition of chiral units (highlighted with cyan connectors) $\downarrow$ The emergence of a glide symmetry means that the infinite system is achiral, yet, it is the boundaries of the finite system that create its chirality (see Fig. $1 \underline{\mathrm{b}}$ ).

The second key feature of a ratchet and pawl is its asymmetric potential. This is formed by a combination of the spring in the pawl mechanism and the asymmetric teeth of the ratchet wheel (Fig.1c). The analogue in the micro-magnetic array is to be found in the potential for the demagnetising fields that emanate from the chiral boundaries of the sample. If the array is magnetised by an applied field such that all the magnetic elements point "up" (as in Fig. 1) and the field is removed, then the magnetisation of the array will thermally relax to eliminate these stray fields and hence minimise the energy stored within them. Calculations confirm that the chirality of the sample boundary is reflected by highly asymmetric energy barriers to rotational motion of the magnetization of individual elements [3]. Thus, when the field is removed, the magnetization of the individual elements, as well as that of the whole array, tends to turn one-way and not the other (Fig. 1d). A small bias field can then reverse the initial array magnetisation, having involved motion in only one direction.

The discovery of Gliga and colleagues, is rather remarkable: it is relatively straightforward to introduce chirality into a micro-magnetic array, but it is much harder to design an asymmetric potential that mimics the pawl and teeth of the mechanical ratchet. The researchers note the importance of "antivortex" textures in the simulated stray field: in two dimensions these are stable topological defects and their rearrangement appears to correlate with asymmetry of the potential that is responsible for the ratchet action. The fact that this ratchet rotates by thermal relaxation, rather than by mechanical driving, gives it a unique aspect that distinguishes it from other approaches to the magnetic ratchet $[4,5]$.

To see ratchet action in a thermally evolving system is somewhat disconcerting at first sight, as it reminds one of a Brownian ratchet (a ratchet driven by Brownian motion). This is considered to be | impossible in the absence of temperature gradients because ${ }_{2}$ near to equilibrium, thermal fluctuations of the pawl mechanism nullify the ratchet action [6]. The micro-magnetic array is not,
Martiradonna, Luigi 5/9/2017 15:04

Comment [4]: I think the use of bold letters is very clear, I would keep it as is. Once the draft is finalized, I will discuss with our copy-editors if this is against our style, and if they think there are better alternatives. You will see their suggestions at the proofs stage.

\section{Martiradonna, Luigi 5/9/2017 15:04}

Comment [5]: In the new figure, I am suggesting to remove the representation as ratchet wheels.

Also, I would avoid showing a 'ratchet wheel' in the figure, as this may confuse the reader - we may expect that the whole 'wheels' structurally rotate in the array, whereas it is the magnetization that rotates while keeping the structure unchanged, as you explain later.

Martiradonna, Luigi 5/9/2017 13:03

Deleted: - fittingly, we can choose these to look like four-toothed ratchet wheels

Martiradonna, Luigi 5/9/2017 13:04

Deleted: However $\mathrm{t}$

Martiradonna, Luigi 5/9/2017 13:04

Deleted: . As in the example above (and indeed as in a mechanical ratchet wheel),

\section{Martiradonna, Luigi 5/9/2017 15:04}

Comment [6]: Here we may refer to the 'ratchet and pawl' sketch, to be included in the figure (see below)

Martiradonna, Luigi 5/9/2017 14:31

Deleted: that of the illustrated array turns clockwise

Martiradonna, Luigi 5/9/2017 15:04

Comment [7]: The magnetic rotation should correspond to the 'dynamic ratchet' mentioned by the authors - I would suggest making this explicit in the figure, by adding a panel that shows the oneway rotation of the magnetic field (similar to Fig.1 from the paper, which I have pasted below but that may possibly be redrawn using the dumbbell graphics you proposed for the other panels). If you think there is a simple way to do it, you may also show in this panel what is acting as 'pawl' is the array: Is it the external magnetic field, or the arrangement of stray fields, or antivortex textures, or the boundaries themselves?

Given the quite extensive discussion on the role of boundaries in the first part of the N\&V, it would be great if panel $d$ could show (possibly in a simple way) what is the role of boundaries and chirality in creating this magnetic ratchet effect.

Martiradonna, Luigi 5/9/2017 14:30

Deleted: et al

Martiradonna, Luigi 5/9/2017 14:30

Deleted:

Martiradonna, Luigi 5/9/2017 14:31

Deleted: Gliga et al. 
of course, a Brownian ratchet, because the free energy of the system is minimised during the demagnetisation process. The resulting asymmetric dynamics may not be surprising in principle for a magnet [7], but to preserve them to this extent in a complex interacting system is most unusual.

As a meta-material, the micro-magnetic array of Gliga and coworkers is in the "artificial spin ice" family [2]. Important recent developments [8,9] have brought such arrays much closer to realising the precise and beautiful physics of the real spin ice materials. Alongside earlier work on magnetotransport [10], the conclusions of this study [3] showcase the importance of chirality, topology and boundaries in two dimensions and how these may be used to introduce functionality. Admittedly the newly discovered magnetic ratchet is more of a "trick" than anything obviously useful_the rotation process (requires up to several hours, But as the long and rich history of magnetism has amply demonstrated, today's tricks become tomorrow's technology. In this sense, artificial spin ice and its relatives hold much promise for the future.

Steven T. Bramwell is at the University College London, London Centre for Nanotechnology and Department of Physics and Astronomy, 17-19 Gordon Street London WC1H OAJ, U. K.

mail: s.t.bramwell@ucl.ac.uk

\section{References}

[1] Ewing, J. A. Contributions to the Molecular Theory of Induced Magnetism. Proc. R. Soc. Lond., 48, 342-358 (1890).

[2] Nisoli, C., Moessner, R. \& Schiffer, P. Artificial spin ice: Designing and imaging magnetic frustration. Rev. Mod. Phys. 85, 1473-1490 (2013).

[3] Gliga S., Hrkac, G., Donnelly, C., Büchi, J., Kleibert, A., Cui, J., Farhan, A., Kirk, E., Chopdekar, R. V., Masaki, Y., Bingham, N. S., Scholl, A., Stamps, R. L., \& Heyderman, L. J. Emergent dynamic chirality in a thermally driven artificial spin ratchet. Nature Materials (to be completed)

[4] Franken, J. H., Swagten, H. J. M.\& Koopmans, B. Shift registers based on magnetic domain wall ratchets with perpendicular anisotropy. Nature Nanotechnology 7, 499-503 (2012).

[5] Magnetic ratchet for three-dimensional spintronic memory and logic.

Lavrijsen1, R., Lee, J. H., Fernández-Pacheco, A., Petit, D. C. M. C., Mansell, R. \& Cowburn, R. P. Nature 493, 647-50 (2013).

[6] Feynman, R. P. The Feynman Lectures on Physics vol. 1, ch. 46 (Addison-Wesley, Reading, MA, 1963).

[7] Onsager, L. Reciprocal relations in irreversible processes I. Phys. Rev. 37 405-426 (1931)

[8] Perrin, Y., Canals B. \& Rougemaille, N. Extensive degeneracy, Coulomb phase and magnetic monopoles in artificial square ice. Nature 540, 410-413 (2016).

\begin{tabular}{|c|}
\hline $\begin{array}{l}\text { Comment [8]: I think this sentence is fine, given } \\
\text { that you have also added a reference to the } \\
\text { Onsager paper here. }\end{array}$ \\
\hline Martiradonna, Luigi 5/9/2017 14:40 \\
\hline Deleted: et al. \\
\hline Martiradonna, Luigi 5/9/2017 14:40 \\
\hline Deleted: Gliga et al. \\
\hline Martiradonna, Luigi 5/9/2017 14:49 \\
\hline Deleted: , \\
\hline Martiradonna, Luigi 5/9/2017 15:04 \\
\hline $\begin{array}{l}\text { Comment [9]: This sentence seems a bit too } \\
\text { informal. }\end{array}$ \\
\hline Martiradonna, Luigi 5/9/2017 14:46 \\
\hline $\begin{array}{l}\text { Deleted: and it is a slow trick at that (to } \\
\text { observe the rotation of the ratchet takes the best } \\
\text { part of a day }\end{array}$ \\
\hline Martiradonna, Luigi 5/9/2017 14:49 \\
\hline Deleted: ) \\
\hline
\end{tabular}


[9] Östman, E., Stopfel, H., Chioar, J. A., Arnalds, U. B., Stein, A., Kapaklis, V. \& Hjörvarsson, B. The importance of the weak: Interaction modifiers in artificial spin ices. arXiv:1706.02127 [cond-mat.mes-hall] (2017).

[10] Branford, W. R., Ladak, S. Read, D. E., Zeissler, K. \& Cohen, L. F. Emerging Chirality in Artificial Spin Ice. Science 35 , 1597-1599 (2012). a

b

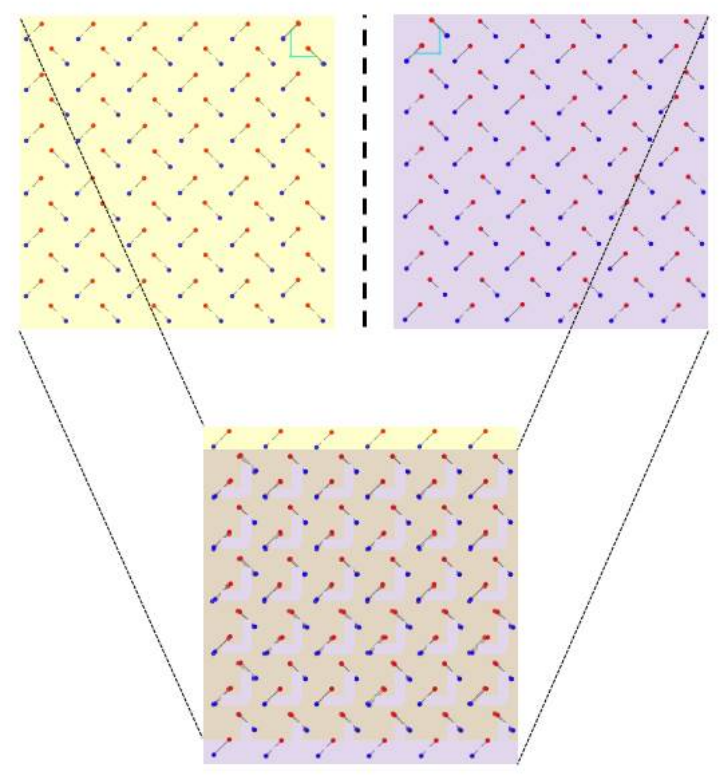

C

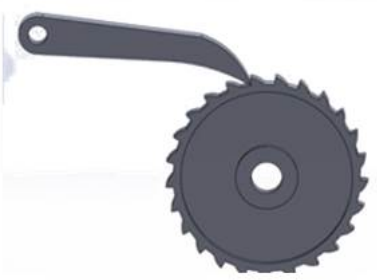

d

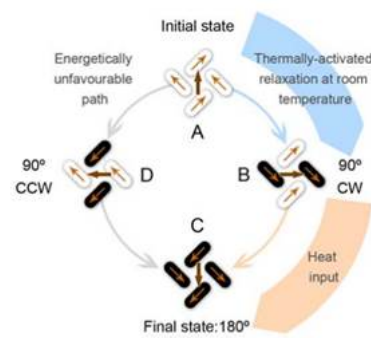

Figure 1| A magnetic ratchet in an artificial spin ice, a. Schematic of a small portion of the magnetic array of Ref. [3], which consists of submicron magnetic needles ( $\mathrm{red}=$ south pole, blue $=$ north pole)

| fixed in a specific pattern. The array on the left (yellow background) is shown in the fully magnetised state, formed by applying a magnetic field in the "upward" direction. The cyan lines are an aid to the eye to indicate the smallest "asymmetric unit" of the structure. The array on the right (purple background) js its mirror image formed by reflection in the vertical dashed line (where the magnets have been treated as dipoles in the mirror operation). $\mathbf{b}$. The array and its mirror image shifted vertically and superimposed. The two may be brought into coincidence everywhere except on the top and bottom boundaries, Because of this glide symmetry, the infinite array is achiral but the finite array remains chiral by virtue of its boundaries. c. a sketch of a ratchet and pawl. $d$. The ratchet effect in the magnetic array....
Comment [10]: This is fine, but we will only be able to use the ArXiv reference if the paper has not been assigned a NPhys DOI in time.

Martiradonna, Luigi 5/9/2017 15:04

Comment [11]: Ok? We need a small sentence

introducing the whole figure.

Martiradonna, Luigi 5/9/2017 14:57

Deleted: (a)

Martiradonna, Luigi 5/9/2017 14:57

Formatted: Font:Bold

Martiradonna, Luigi 5/9/2017 14:56

Deleted: (b)

Martiradonna, Luigi 5/9/2017 14:57

Deleted: and

Martiradonna, Luigi 5/9/2017 14:57

Deleted: The array has been decorated with circles to illustrate the local chirality or sense of rotation, which changes sign on reflection. (c)

Martiradonna, Luigi 5/9/2017 14:57

Formatted: Font:Bold

Martiradonna, Luigi 5/9/2017 14:58

Deleted: (red, yellow strips)

Martiradonna, Luigi 5/9/2017 15:03

Formatted: Font:Bold

Martiradonna, Luigi 5/9/2017 15:03

Formatted: Font:Bold

Martiradonna, Luigi 5/9/2017 15:04

Comment [12]: This caption should be updated according to the content of the panel

Martiradonna, Luigi 5/9/2017 15:03

Deleted: The potential of the magnetic fields emanating from these chiral boundaries mimics the asymmetric potential of a ratchet and pawl mechanism. 
